\title{
Enrichment of Female Germline Stem Cells from Mouse Ovaries Using the Differential Adhesion Method
}

\author{
Meng Wu Jiaqiang Xiong ${ }^{\mathrm{a}} \quad$ Lingwei Ma $^{\mathrm{a}}$ Zhiyong Lu ${ }^{\mathrm{b}} \quad$ Xian Qin ${ }^{\mathrm{b}} \quad$ Aiyue Luo $^{\mathrm{a}}$ \\ Jinjin Zhang ${ }^{a}$ Huan Xiec ${ }^{c}$ Wei Shen ${ }^{a}$ Shixuan Wanga \\ aDepartment of Obstetrics and Gynecology, Tongji Hospital, Tongji Medical College, Huazhong

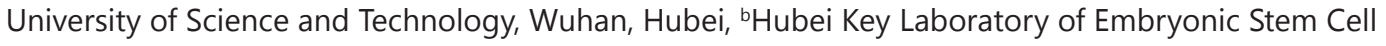 \\ Research, Tai-He Hospital, Hubei University of Medicine, Shiyan, Hubei, 'Department of Obstetrics and \\ Gynecology, Second people's Hospital, Yichang, Hubei, China.
}

\section{Key Words}

Female germline stem cells • Differential adhesion method $\cdot$ AKT pathway $•$ Self-renewal

\begin{abstract}
Background/Aims: The isolation and establishment of female germline stem cells (FGSCs) is controversial because of questions regarding the reliability and stability of the isolation method using antibody targeting mouse vasa homologue $(\mathrm{MVH})$, and the molecular mechanism of FGSCs self-renewal remains unclear. Thus, there needs to be a simple and reliable method for sorting FGSCs to study them. Methods: We applied the differential adhesion method to enrich FGSCs (DA-FGSCs) from mouse ovaries. Through four rounds of purification and 7-9 subsequent passages, DA-FGSC lines were established. In addition, we assessed the role of the phosphoinositide-3 kinase (PI3K)-AKT pathway in regulating FGSC self-renewal. Results: The obtained DA-FGSCs spontaneously differentiated into oocyte-like cells in vitro and formed functional eggs in vivo that were fertilized and produced healthy offspring. AKT was rapidly phosphorylated when the proliferation rate of FGSCs increased after 10 passages, and the addition of a chemical PI3K inhibitor prevented FGSCs self-renewal. Furthermore, overexpression of AKT-induced proliferation and differentiation of FGSCs, c-Myc, Oct-4 and Gdf-9 levels were increased. Conclusions: The differential adhesion method provides a more feasible approach and is an easier procedure to establish FGSC lines than traditional methods. The AKT pathway plays an important role in regulation of the proliferation and maintenance of FGSCs. These findings could help promote stem cell studies and provide a better understanding of causes of ovarian infertility, thereby providing potential treatments for infertility.
\end{abstract}




\section{Introduction}

The concept that oogenesis continues into reproductive life has been well-established in non-mammalian species [1,2], and it is widely accepted in mammals that mature oocytes are supplied from a pool of primordial follicles in the adult ovary without the potential for renewal [3-5]. However, recent studies have indicated that the formation of oocytes is not, as traditionally believed, restricted to the foetal or perinatal period [6, 7]. Studies have confirmed that FGSCs are capable of generating functional eggs that can be fertilized and produce offspring in mice [8-10]. In 2009, Zou et al. successfully isolated and purified FGSCs from neonatal and adult mouse ovaries via MACS (magnetic activated cell sorting) using a germline marker named MVH (mouse vasa homologue, also commonly referred to as DEAD box polypeptide 4 or $\mathrm{Ddx} 4$ ) for the first time [8]. Four years later, White et al. first isolated and purified hFGSCs (human Female Germline Stem Cells) from human ovaries of adults of reproductive age using FACS (fluorescence-activated cell sorter) with the same marker Zou et al. used [11]. In 2015, our group also isolated FGSCs from mouse ovaries via MACS (MACSFGSCs) [12,13]. In 2016, Wu's group also successfully isolated hFGSCs from scarce ovarian cortical tissues that were present in follicular aspirates [14]. However, several groups were unable to isolate FGSCs [15-17], which led them to cast doubt on the existence of the stem cells. For example, Hernandez et al. claimed that cells isolated using the anti-Ddx4 antibody showed no evidence of Ddx4 mRNA or protein expression and were unable to show new oocyte formation in transplantation-based assays [18]. Larissa et al. reported that FACSsorted putative oogonial stem cells from the ovary are neither Ddx4-positive cells nor germ cells [19]. Lei et al. and Zhang et al. confirmed that there was no re-establishment of the follicle pool by adult germ cells under physiological or pathological conditions [20,21]. However, in 2016, Guo's study confirmed that germline stem cells are active in postnatal mouse ovaries under physiological conditions. These researchers also reported that isolation of FGSCs with antibody targeting Ddx4 was questionable, and their work proposed that Oct-4+Dazl- but not Oct-4+Dazl+ or Oct-4+Ddx4+ cells display mitotic proliferation [22]. This finding is not in keeping with the previous studies that reported the isolation of FGSCs with Ddx4 antibody $[8,11]$. Therefore, the underlying reasons for the controversy are technical, rather than due to an absence of FGSCs in mammalian ovaries. According to our experience, we have speculated that the main reasons why FGSCs remain poorly studied may include the following factors: the small number of FGSCs in ovaries; the nonspecific isolation marker; the complexity of the isolation procedure and the unknown culture conditions. At present, although Ddx4 and Fragilis (also commonly referred to as Ifitm3, interferon-induced transmembrane protein 3 ) are the most frequently used biomarkers to isolate FGSCs using MACS or FACS methods, and there is no internationally accepted method or specific biomarker due to the dispute between proponents and opponents of FGSCs.

The differential adhesion method has successfully been applied in the purification of many other types of cells, such as mesenchymal stem cells, neural stem cells and spermatogonial stem cells [23-25]. According to our experience and Wu's study [26], the speed of somatic cell attachment to a plate is faster than that of FGSCs; thus, it may be feasible to isolate FGSCs using the differential adhesion method. Although the differential adhesion method has been recently reported to be used to isolate FGSCs, a stable cell line has not been constructed, and there was insufficient evidence for FGSC identification in that article [27]. In our study, we attempted to use the differential adhesion method to facilitate acquirement of FGSCs. This method offers the relative advantage of a rapid and simple procedure to obtain an FGSC line. In addition, we found that the proliferation rate of FGSCs increased after a period of culture, especially after 10 passages. Next, we investigated the molecular mechanisms that control the proliferation of FGSCs and found that the AKT pathway mediates self-renewal of FGSCs. Previous studies have shown that the PI3K-AKT pathway was strongly observed in the mRNAs and lncRNAs of FGSCs [28]. It is known that the AKT pathway is involved in self-renewal of spermatogonial stem cells, but little is known regarding the effects of the AKT pathway on self-renewal of FGSCs.

\section{KARGER}




\section{Cellular Physiology Cell Physiol Biochem 2018;46:2114-2126 and Biochemistry Published online: May 03, $2018 \quad \begin{aligned} & \text { DOI: 10.1159/000489452 } \\ & \begin{array}{l}\text { c } 2018 \text { The Author(s). Published by S. Karger AG, Basel } \\ \text { www.karger.com/cpb }\end{array}\end{aligned}$ \\ Wu et al.: Enrichment of Female Germline Stem Cells}

\section{Materials and Methods}

\section{Animals}

Mice (C57BL/6) were purchased from the Centre of Medical Experimental Animals of Hubei Province (Wuhan, China). All procedures for animal experiments were approved by the Animal Care and The Committee of Tongji Medical College at the Huazhong University of Science and Technology in China and were carried out in accordance with the National Research Council Guide for Care.

\section{Isolation and culture of DA-FGSCS}

DA-FGSCs were isolated using the methods described below. In brief, for each isolation, eight ovaries were dissected from four 6-week-old mice (7-day-old and 12-week-old mice were also used to isolate DAFGSCs) and then were cut into a slurry in Hank's balanced salt solution without calcium or magnesium (HBSS BOSTER) containing collagenase/Dnase I (Type IV; Sigma), followed by incubation at $37^{\circ} \mathrm{C}$ with intermittent shaking for $15 \mathrm{~min}$. Then, undigested ovarian debris was removed, and the dissociated cells were centrifuged at $300 \mathrm{~g}$ for $5 \mathrm{~min}$ to remove the collagenase and then washed once with FGSC culture medium. Finally, the cells were seeded into one well of a 6-well plate without a feeder layer. The cells were cultured with the defined medium, which consisted of minimum essential medium $\alpha$ (MEM- $\alpha$ ), 10\% FBS (Gibco), 1000 units/ml LIF (leukaemia inhibitory factor; Millipore), $1 \mathrm{ng} / \mathrm{ml} \mathrm{bFGF} \mathrm{(basic} \mathrm{fibroblast} \mathrm{growth}$ factor; BD Biosciences), $10 \mathrm{ng} / \mathrm{ml} \mathrm{EGF} \mathrm{(mouse} \mathrm{epidermal} \mathrm{growth} \mathrm{factor;} \mathrm{Sigma),} 20 \mathrm{ng} / \mathrm{ml}$ human GDNF (glial cell line-derived neurotrophic factor; R\&D systems), $1 \mathrm{mM}$ non-essential amino acids, $1 \mathrm{mM}$ sodium pyruvate, $0.1 \mathrm{mM} \beta$-mercaptoethanol, $1 \times$ concentrated N2-supplement, and $1 \times$ concentrated penicillinstreptomycin. LY294002 was used at $20 \mu \mathrm{M}$. When the cells reached confluence on the six-well plate, they were digested with trypsin followed by neutralization by adding $10 \%$ foetal bovine serum (FBS). Next, the digested cells were planted in one well of a six-well plate with gelatin for differential adherence selection, and after 30 minutes cultured at $37^{\circ} \mathrm{C}$, the supernatant that contained cells unattached to the six-well plate was collected and transferred to one well of a 24-well plate for culture without feeder cells. When the cells reached confluence again within the 24-well plate, they were digested and transferred to one well of a 6-well plate, and 30 minutes later, the supernatant was gently collected and transferred to the 24-well plate again. After four rounds of purification, and the remaining cells only required repeated digestion when they reached confluence. Within 7-9 passages, the somatic cells gradually died, small round cells slowly occupied the majority proportion of the total cells, and finally, the DA-FGSCs line was stably established. The culture plates were checked the next morning after subculture, and the medium was changed if many floating cell fragments were observed. Otherwise, the medium was changed every 2-3 days, and all cultures were maintained at $37^{\circ} \mathrm{C}$ in a $5 \% \mathrm{CO}_{2}$ atmosphere.

\section{Immunofluorescence}

To test whether the DA-FGSCs expressed germ cell-specific genes, immunocytochemistry was conducted as follows: the cells were fixed in 4\% paraformaldehyde for $15 \mathrm{~min}$ at room temperature, and after being washed in PBS three times, the cells were blocked with $3 \%$ bovine serum albumin (BSA) at $37^{\circ} \mathrm{C}$ for $1 \mathrm{~h}$ and incubated overnight at $4^{\circ} \mathrm{C}$ with the following primary antibodies: rabbit polyclonal anti-MVH (1:100 dilution, Abcam), rabbit polyclonal anti-Fragilis (1: 500 dilution, Abcam), anti-Zp3 (1:100 dilution, Santa Cruz Biotechnology), anti-Gdf9 (1:100 dilution, Santa Cruz Biotechnology), anti-Oct-4 (1: 200 dilution, Abcam), and anti-Dppa3 (1:200 dilution, Abcam). After being washed in PBS three times, the cells were subsequently incubated with FITC-conjugated secondary antibodies at 1:200 dilution followed by Hoechst staining for $15 \mathrm{~min}$ at room temperature in the dark. Finally, the cells were visualized under a fluorescence microscope. The STO (derived from mouse SIM embryonic fibroblasts) were used as negative control.

\section{Reverse transcription-polymerase chain reaction and real time-polymerase chain reaction}

Total RNA was extracted separately from ovaries and DA-FGSCs using Trizol reagent (Qiagen) according to the manufacturer's instructions. Approximately $2 \mu \mathrm{g}$ of RNA was treated with Dnase I (Thermo Scientific) to remove trace amounts of DNA contamination, and the RNA was used to synthesize cDNA using transcriptor reverse transcriptase (Takara) based on the manufacturer's instructions. Specific primer pairs targeting Nobox, Zp3, Gdf9, Fragilis, MVH, Tert, Dppa3, Dazl, Prdm1, Oct-4, Ybx2, Sycp3, Dmc1, PCNA, c-Myc and Gapdh were used for PCR reactions. 


\section{Cellular Physiology Cell Physiol Biochem 2018;46:2114-2126 and Biochemistry Published online: May 03, $2018 \quad \begin{aligned} & \text { DOI: 10.1159/000489452 } \\ & \begin{array}{l}\text { c } 2018 \text { The Author(s). Published by S. Karger AG, Basel } \\ \text { www.karger.com/cpb }\end{array}\end{aligned}$}

Wu et al.: Enrichment of Female Germline Stem Cells

Karyotype analysis of DA-FGSCs

After being cultured for more than 20 passages, DA-FGSCs in the logarithmic growth phase were treated with $80 \mathrm{ng} / \mathrm{ml}$ colchicine in FGSC culture medium for $3 \mathrm{~h}$, followed by treatment with $40 \mathrm{mM} \mathrm{KCl}$ for $30 \mathrm{~min}$; then, they were fixed in methanol-acetic acid (3:1), air-dried, stained with Giemsa buffer and finally viewed under a microscope with an oil objective.

Lentiviral transfection of DA-FGSCs and transplantation of GFP-FGSCS

When DA-FGSCs were seeded in one well of a six-well plate and reached the logarithmic phase, the cells were infected with CMV-EGFP virus (Genomeditech) according to the company's manual. Two days later, the expression of GFP was observed under a fluorescence microscope, and when the cells reached confluence, the GFP-labelled DA-FGSCs were purified by FACS. After subculture for several passages, the purified and GFP-labelled DA-FGSCs were transplanted into ovaries of 7-week-old C57/BL female mice. As a control, the filtered supernatant of DA-FGSC culture medium and GFP-STO cells were injected into ovaries of the same type of female mice described above, and each group had 15 mice. In brief, recipients were anaesthetized with $1 \%$ pentobarbitone sodium via intraperitoneal injection, and then, a single-cell suspension of $3 \mu \mathrm{l}$ containing approximately $2 \times 10^{\wedge} 4$ cells passaged 25 generations was injected into each ovary of the recipients using a 10- $\mu$ l NanoFil syringe with a 33-gauge bevelled needle (World Precision Instruments). Thirty-five days after transplantation, the above female mice were mated with adult male mice at 2:1 ratio.

\section{Immunohistochemistry}

The ovarian tissue sections were fixed and processed for immunohistochemical detection of GFP using a rabbit antibody against GFP (1:100 dilution, ProteinTech) along with a Biotin-Streptavidin HRP Detection System (Qiagen) according to the manufacturer's instructions. Ovaries from medium-transplanted and GFPSTO-transplanted female mice served as negative controls for GFP detection. Images were obtained using a Leica microscope.

\section{Ploidy analysis}

Cultured DA-FGSCs (72 h after passage) were collected by trypsinization and washed in ice-cold PBS. After fixation with ice-cold $70 \%$ ethanol at $-20^{\circ} \mathrm{C}$ overnight, cells were washed in PBS and incubated with $0.2 \mathrm{mg} \mathrm{ml}^{-1}$ RNase-A for $1 \mathrm{~h}$ at $37^{\circ} \mathrm{C}$. Propidium iodide was then added $\left(10 \mu \mathrm{g} \mathrm{m}^{-1}\right.$ final), and ploidy status was detected using a BD FACSCalibur flow cytometer. STO cells were used as the control in this experiment.

\section{Statistics}

All experiments were independently replicated at least three times. Quantitative data (means \pm s.e.m. of combined results) were analysed with one-way ANOVA using Statistical Package for the Social Sciences (SPSS) software. Values of $\mathrm{p}<0.05$ were considered to be statistically significant.

\section{Results}

\section{Isolation and purification of DA-FGSCs using differential adhesion}

Through sufficient physical cutting and collagenase digestion of eight ovaries from 6-week-old mice, we obtained a disperse cell solution, which contained granulose cells, theca cells, oocytes and a small number of FGSCs (Fig. 1A-a). After culture for 3 days, the density of the above cells reached confluence and some small colonies formed, which resembled FGSCs in terms of morphology (Fig. 1A-b). Through the first purification in which the supernatant was collected, we obtained a small number of putative FGSCs (Fig. 1A-c). When the density of the first purified cells reached confluence again in a 24-well plate, they were digested, the supernatant was collected again, and a higher purity of FGSCs was obtained after the second purification (Fig. 1A-d). Through four rounds of purification and several subsequent passages, we obtained a DA-FGSC line with a morphology that is similar to that of FGSCs (Fig. 1A-e and 1A-f), exhibiting large cell bodies with little cytoplasm and a large ratio of nuclear plasma as previously described [8]. After the first round of purification, we tested the stemness of the purified cells using ALP (alkaline phosphatase) staining, and 
Fig. 1. Isolation and purification of FGSCs. (A) The FGSCs purification process through differential adhesion. After digestions by collagenase IV, dispersed ovary cells were cultured and selected by 4 rounds of differential adherence selections in gelatin coated wells. Within 7-9 passages, the DAFGSCs line was stably established. (a) The morphology of all the dispersed cells on the second day after digestion of ovaries, including follicles, somatic cells, and a few FGSCs. (b) Small cluster clones of putative FGSCs formed on the third day of cultivation. (c) A small number of putative FGSCs were obtained after the first purification using the differential adhesion method. (d) Higher purity and quantity of FGSCs were obtained after the second purification. (e, f) Within the following subculture after the second purification, the FGSCs became relatively purified and finally were stably established.

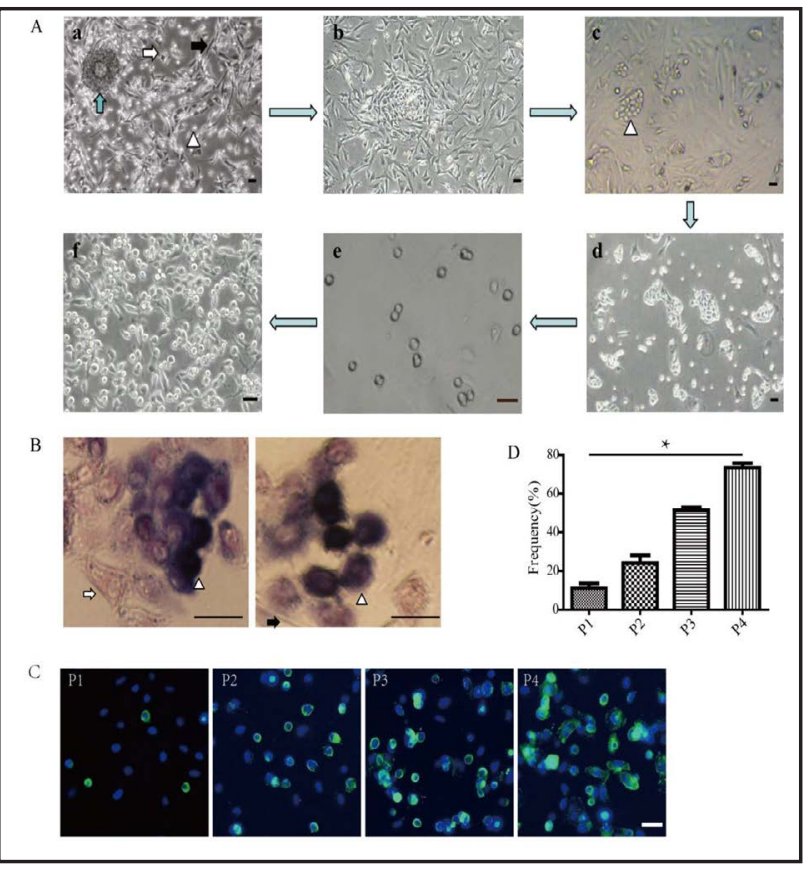
Granulosa cells $\Leftrightarrow \Leftrightarrow$ (white)), theca cells $(\Leftrightarrow$ (black)), follicle ( $\Rightarrow$ (blue)), the putative FGSCs $(\Delta)$. (B) Alkaline phosphatase staining of the cell clusters. (C) Immunofluorescence of MVH (green) during FGSC enrichment. (D) The percentage of cells showing MVH staining. Scale bars: $25 \mu \mathrm{m}(\mathrm{A}-\mathrm{C})$.

Fig. 2. Characteristics of the established DA-FGSCs. (A) Dual immunofluorescence of MVH/EDU and Fragilis/EDU staining in established DA-FGSCs. (B) Immunofluorescence detection of Oct-4 and Dppa3. STO cells were used as the negative control (NC). (C) PCR analysis to determine the gene expression profile of DA-FGSCs. M, 100 bp DNA marker; lane 1, ovary; lane 2, DA-FGSCs; lane 3, NO-RT (PCR of RNA samples without reverse transcription). (D) Cytogenetic analysis of DA-FGSCs. (E) Alkaline phosphatase staining of DA-FGSCs; STO, negative control cells. (F) OLCs formed during DA-FGSC culture. (G) Immunofluorescence analysis of OLCs with antibodies against Gdf-9 and Zp3. (H) FACSbased ploidy analysis of cultured DA-FGSCs. Scale bars: $25 \mu \mathrm{m}(\mathrm{A}, \mathrm{B}, \mathrm{E}), 50 \mu \mathrm{m}(\mathrm{F}, \mathrm{H})$.

the results showed that most of the clonal cells were positive, with a dark blue colour. However, the granulosa cells and theca cells were negative (Fig. 1B). Furthermore, to have an idea of the positive rate of FGSCs during different stages of enrichment at the early stage, we also performed a statistical assessment of the purity of FGSCs from

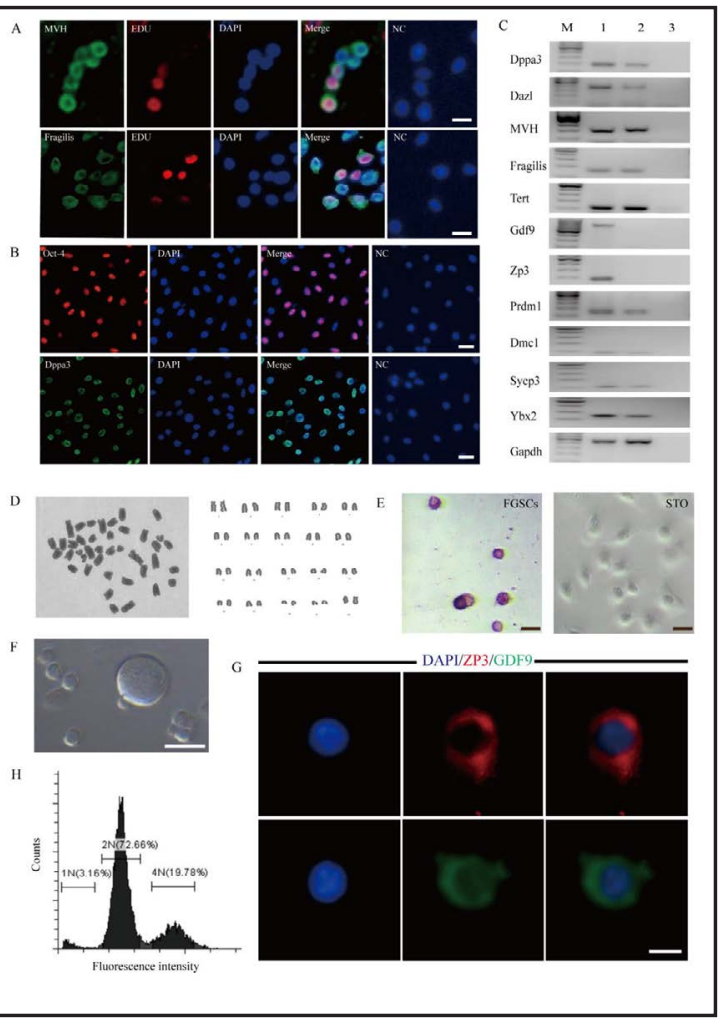
the first round of purification to the fourth round of purification by examining MVH immunofluorescence (Fig. 1C and Fig. 1D). 


\section{Cellular Physiology Cell Physiol Biochem 2018;46:2114-2126 and Biochemistry Published online: May 03, $2018 \quad \begin{aligned} & \text { DOI: 10.1159/000489452 } 2018 \text { The Author(s). Published by S. Karger AG, Basel } \\ & \text { www.karger.com/cpb }\end{aligned}$ Wu et al.: Enrichment of Female Germline Stem Cells}

Identification of DA-FGSCS using immunofluorescence, gene expression, ALP staining, karyotyping and cell differentiation

After stable establishment of DA-FGSCs, immunofluorescence assays showed that some DAFGSCs were positive for both MVH/EDU and Fragilis/EDU (Fig. 2A). To confirm the identity of DAFGSCs, stemness-related genes, such as Oct-4 and Dppa3, were also examined by immunofluorescence, and the results showed that Oct4 and Dppa3 were expressed on DA-FGSCs (Fig. 2B). Next, the PCR detection showed that the DA-FGSCs expressed the germ cell genes Fragilis, MVH, Tert (telomerase reverse transcriptase), Dppa3, Dazl (a germ cell-specific RNA-binding protein), Prdm1 (PR domain containing 1 with ZNF domain), Dmc1, Sycp3, and Ybx2 and do not express oocytespecific genes (Zp3 and Gdf9); the ovaries were all positive for the above genes as the positive control, PCR of RNA samples without reverse transcription were used as the negative control (Fig. 2C). Next, cytogenetic analysis showed that most FGSCs had a normal karyotype with twenty pairs of chromosomes (Fig. 2D). Meanwhile, alkaline phosphatase, another protein expressed by FGSCs, was detected, and the results showed that the DA-FGSCs were positive for ALP staining. STO cells was used as the negative as control (Fig. 2E). In addition, a small number of DA-FGSCs can spontaneously differentiate into OLCs (oocyte-like cells) (Fig. 2F), and this phenomenon was also reported by Pacchiarotti et al [29] and White et al [11]. Furthermore, to confirm the characteristics of OLCs, the oocytespecific expression of the proteins Zp3 and Gdf9 was detected by immunofluorescence, and the results revealed that certain of the OLCs expressed Zp3 and Gdf9 (Fig. 2G). Finally, to illuminate whether these OLCs are haploid, FACS-based ploidy analysis of cultured DA-FGSCs 72 hours after passage was conducted, and the results showed that $3.16 \%$ of the total cells reached haploid status (Fig. $2 \mathrm{H}$ ).

\section{Transfection and transplantation of DA-FGSCs}

After the DA-FGSCs lines were stably established, the cells that had been passaged for 20 generations were infected with CMV-EGFP lentivirus (Fig. 3A) in a 6-well plate during the logarithmic growth phase. The EGFP-FGSCs were purified using FACS when they reached confluence and 78\% of the cells were GFP positive (Fig. 3B and Fig. 3C). After subculture for two weeks, EGFP-FGSCs were transplanted into ovaries of recipient females (Fig. 3D). Then, 35 days after transplantation, a serial-section immunohistochemistry analysis revealed the presence of GFP-positive oocytes along with host follicles containing GFP-negative oocytes, and we did not detect any GFP-positive oocytes in the ovaries that received mock injections (Fig. 4A). After 55-85 days post-transplantation, 86\% (13/15) of the recipients produced offspring through natural mating with male wild-type C57BL/6 mice (Fig. 4B). The GFP and PURO (puromycin) genes, which were integrated into the genome of DA-FGSCs, were 


\section{Cellular Physiology Cell Physiol Biochem 2018;46:2114-2126 \begin{tabular}{l|l} 
DOI: 10.1159/000489452 & $\begin{array}{l}\text { O 2018 The Author(s). Published by S. Karger AG, Basel } \\
\text { www.karger.com/cpb }\end{array}$
\end{tabular}

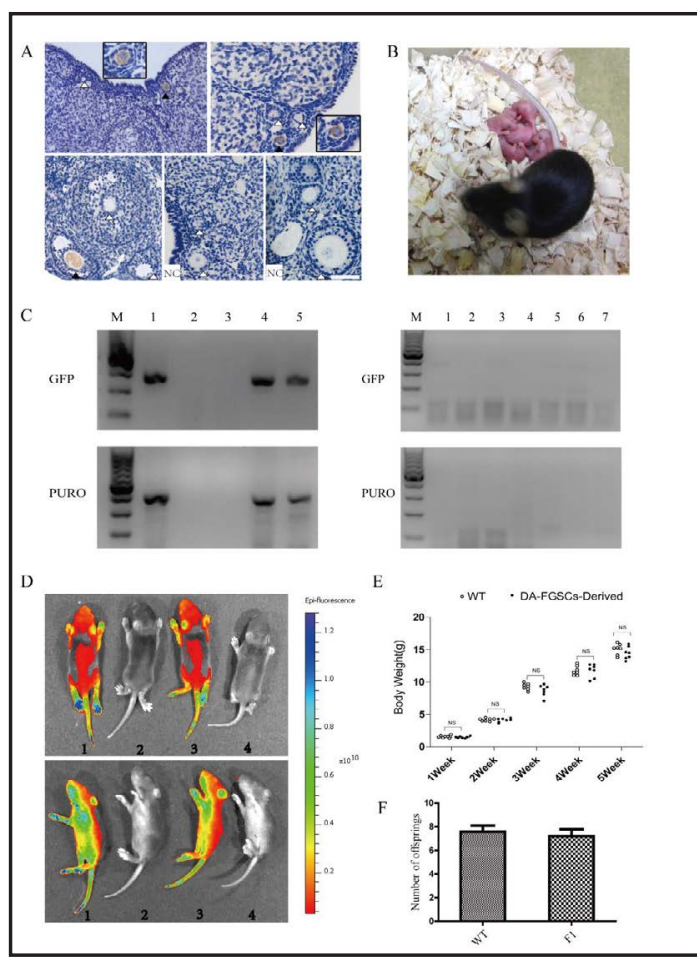

Fig. 4. GFP offspring generated from the transplantation of DA-FGSC. (A) Immunohistochemistry staining of EGFP-positive oocytes (EGFP+, brown) surrounded by smaller EGFP- oocytes in ovaries of wild-type mice injected with GFP-FGSCs. ( $\Delta$ (black))represents GFP-positive oocytes; $(\Delta$ (white))represents GFPnegative oocytes. NC represent the negative control (medium-transplanted group). (B) The offspring originated from the DA-FGSC transplantation group. (C) Identification of GFP and PURO genes from genomic DNA using PCR. Both GFP and PURO genes were detected in some of the offspring of the DA-FGSC transplantation group; Both GFP and PURO genes were not detected in the medium transplantation groups (Right). GFP (366 bp) transcription was examined with the primer set 5' -CGACGGCAACTACAAGACCCG-3' and 5'- ACGAACTCCAGCAGGACCATG-3'; PURO (388 bp) transcription was assessed with the primer set 5'-GTCACCGAGCTGCAAGAACT-3' and 5'-GCTCGTAGAAGGGGAGGTTG-3'. (D) Offspring that inherited the GFP gene were detected under a live-imaging machine. Samples 1 and 3 were GFP positive, and samples 2 and 4 were negative. (E) Development of the body weights of offspring from the recipients and the genetically matched wildtype mice. NS, not significant ( $p>0.05$ ). (F) The F1 generation derived from DA-FGSCs have normal fertility compared with wild-type mice. Scale bars: $50 \mu \mathrm{m}(\mathrm{A})$.

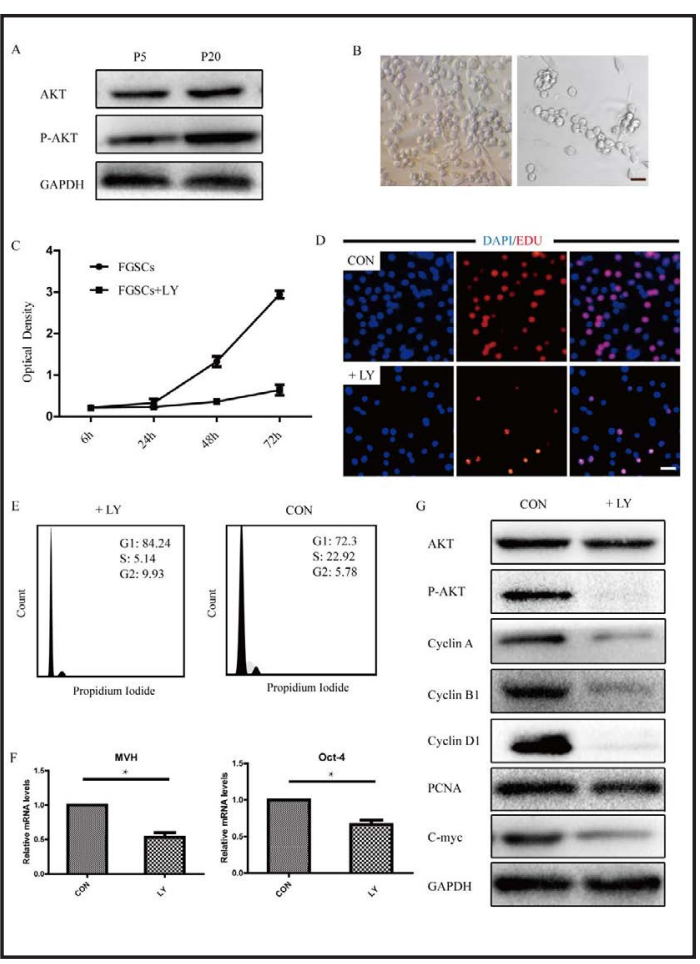

Fig. 5. Inhibition of FGSC proliferation by LY294002. (A) Western blots showed the expression of AKT and phospho-AKT in the cultured FGSC culture after 5 passages and 20 passages. (B) The appearance of FGSCs in the presence of LY294002. (C) The changes in cell proliferation as detected by a CCK-8 assay. (D) The EDU detection of FGSCs in the presence of LY294002. (E) Analysis of cell cycle distribution 3 days after passage in the presence of LY294002. (F) The expression of MVH and Oct-4 analysed via RT-PCR. (G) Expression of proliferation-associated proteins after LY294002 stimulation assessed by western blotting. ${ }^{*} \mathrm{p}<0.05$. Scale bars: $25 \mu \mathrm{m}(\mathrm{B}, \mathrm{D})$.

identified in the genomic DNA of offspring by PCR, and the results showed that 20 of the $82 \mathrm{~F} 1$ offspring were both positive for GFP and PURO genes, whereas no positive samples were detected in the control group (Fig. 4C). In addition, some of the offspring were imaged under a live-imaging machine (Caliper Life Sciences) one week after birth and showed the presence of GFP (Fig. 4D). Both female and male offspring originating from DA-FGSCs were healthy and fertile, and the body weights of offspring genetically matched those of wild-type mice (Fig. 4E). The transgenic mice are 11 months old at the time of the writing of this article. Reciprocal hybridization showed that 
Fig. 6. Development of FGSCs after AKT overexpression in vitro. (A) The change in cell proliferation as detected by CCK-8 assays. (B) The EDU detection of AKT overexpression in FGSCs. (C) The expression of PCNA, c-Myc, cyclin A, MVH, Oct-4 and Gdf-9 analysed by RTPCR. (D) Immunofluorescence analysis for Gdf-9 cells in the AKT-overexpression FGSCs. (E) Percentage of cells showing punctate Gdf9 staining. $* \mathrm{p}<0.05$. Scale bars: $25 \mu \mathrm{m}$ (B, D).

the F1 generation had normal fertility (Fig. $4 F$ ). In conclusion, the above results vigorously verified that DA-FGSCs could differentiate into functional eggs in vivo and finally result in progeny.

AKT pathway
mediates self-
renewal of FGSCS
In the process of
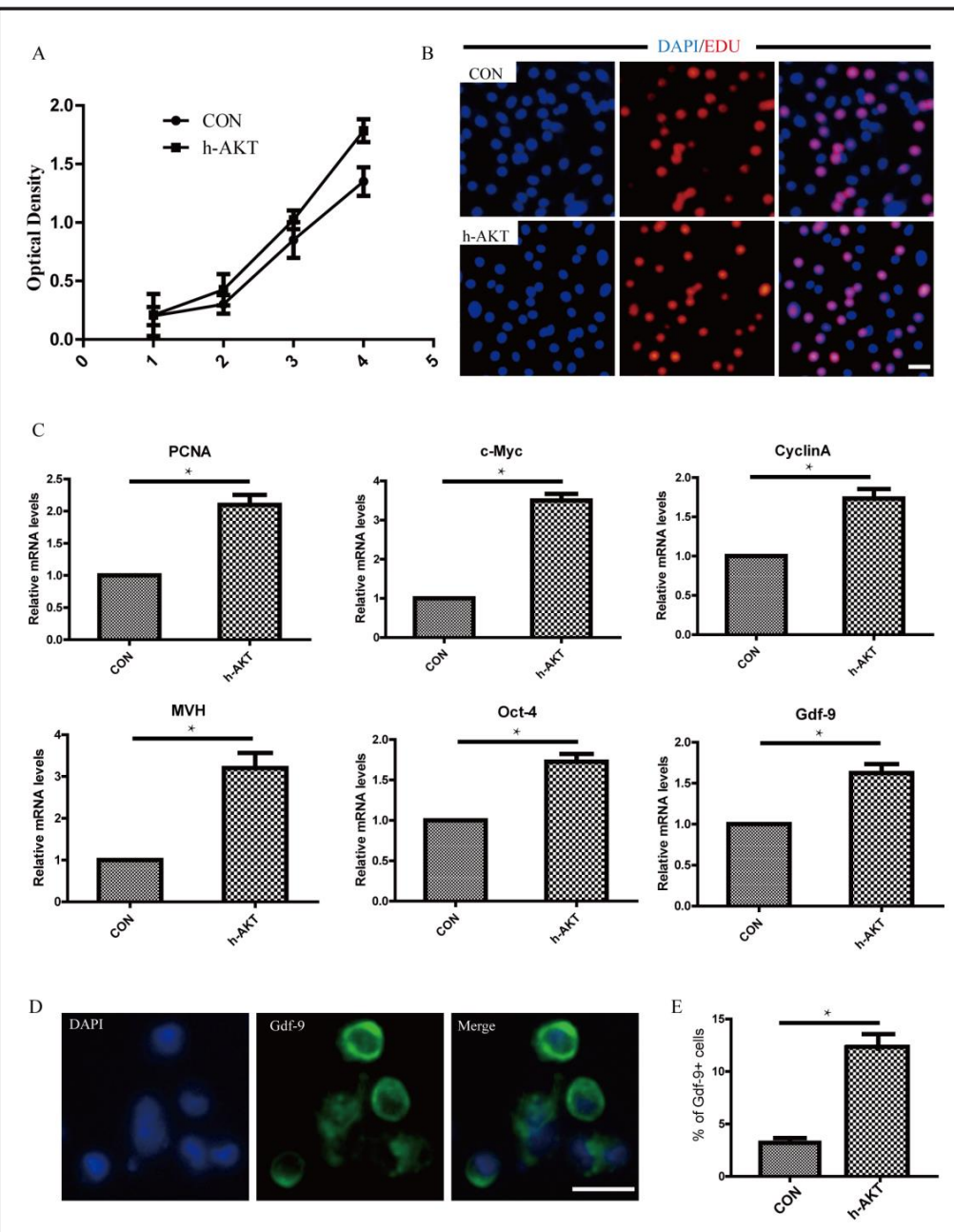

FGSC culture, we observed that the proliferation rate of early generations was slow. After 10 passages, the proliferation rate increased, and FGSCs could be passaged at a 1:3-1:4 split ratio. To help elucidate the molecular mechanisms that control FGSCs proliferation, we further asked whether the AKT signalling pathway was activated in the cultured FGSCs. Western blots clearly revealed that AKT and AKT phosphorylation was increased in the FGSCs cultured for 20 generations compared to those cultured for 5 generations (Fig. 5A). To investigate the AKT signalling pathway in the self-renewal of FGSCs, we first examined the effects of pharmacological inhibitors (LY294002) of the PI3K pathway. When FGSCs were cultured in the presence of LY294002 (Fig. 5B), the growth of FGSCs cells was significantly inhibited, and the number of cells did not increase (Fig. 5C and 5D). Cell cycle analysis revealed that LY294002 treatment increased the proportion of cells in G1 phase (Fig. 5E). In addition, the specific FGSC markers MVH and Oct-4 were slightly less expressed in the LY294002 group cells, further supporting a role for the AKT pathway in differentiation of FGSCs (Fig. 5F). We also examined the expression of cyclin molecules after inhibition of AKT. The Western blot analysis indicated that the expression of c-Myc and cyclin proteins were downregulated in the LY294002-treated group (Fig. 5G).

To confirm the role of the AKT pathway in regulating FGSC proliferation and differentiation, we also transduced cells with AKT-overexpressing lentivirus. The cells overexpressing AKT proliferated slightly faster than wild-type FGSCs (Fig. 6A and 6B). Realtime PCR indicated that the levels of proliferation markers, including PCNA, c-Myc and cyclin A were higher than those in control cells (Fig. 6C). MVH and Oct-4 and the oocyte specificmarker Gdf-9, were also more highly expressed in the AKT overexpression group (Fig. 6C). 
Wu et al.: Enrichment of Female Germline Stem Cells

Immunofluorescence assays showed that more cells expressed Gdf- 9 after AKT activation, the proportion of Gdf- $9^{+}$cells increased from $3.2 \%$ to $12.3 \%$, suggesting that a portion of FGSCs started spontaneous differentiation (Fig. 6D and 6E). The results indicated that the AKT pathway may promote proliferation and differentiation of FGSCs.

\section{Discussion}

Spermatogonial stem cells, which have been widely studied, have been confirmed to form sperm in vitro and in vivo for many years [30, 31]. However, whether FGSCs exist has aroused widespread controversy between supporters and opponents in recent years $[10,19$, $21,32,33]$. In 2009, since Wu et al. first confirmed the presence of FGSCs in mouse ovaries, many other researchers have gradually confirmed the existence of FGSCs in other mammalian species [34-36]. Furthermore, several research groups have extended the application of FGSCs in different fields, such as in vitro differentiation into oocytes [37], generation of transgenic mice [38], mechanism research [39, 40] and cell therapy [41, 42]. Although the supporters are in favour of the existence of FGSCs, there are still a number of opponents. One of the important reasons is that questions about the specificity and reliability of the anti-Ddx4based FGSC-isolation protocol. There are many types of cells in ovaries with complicated growth and differentiation processes. Compared to somatic cells in the ovary, there are relatively few FGSCs $(0.014 \%)$ in ovaries [11], and utilizing an inappropriate molecule to label putative germline stem cells, could lead to failure in isolating FGSCs [10, 19, 22, 34, 43]. Although Wu's group has used Fragilis-Based MACS to improve the efficiency of FGSC purification [44], Fragilis is also present in the oviduct, stromal cells and oocytes, confirming that this germline marker is not germline-specific [19]. Thus, the limiting factor for the study of FGSC behaviour and characteristics in vitro has been separation and purification of these cells while maintaining their self-renewal ability.

In this study, we provide a new methodology that allows efficient enrichment of FGSCs from mouse ovaries through differential adhesion. Based on our experience, we found that the attachment rate of both granulosa cells and stromal cells is faster than that of FGSCs, and thus, we took advantage of the different attachment rate of these cells to isolate FGSCs. Although these enriched cells might be heterogeneous, they contained FGSCs along with other types of cells that function similar to Sertoli cells in the culture of spermatagonial stem cells, providing a niche to promote the growth of stem cells in the early stages. After subculture for several passages, the number of heterogeneous cells, such as granulosa cells and stromal cells, was gradually reduced, and small round cells slowly became the majority proportion of these cells. After four rounds of purification using the differential adhesion method followed by several passages for approximately one month, an FGSC line was stably established, and the FGSCs showed the ability to differentiate into functional eggs and were fertilized to produce offspring, and the offspring were fertile and healthy and remained alive for at least 11 months without any apparent abnormality. Based on the above results, we maintain that the cells isolated by the differential adhesion method were primarily FGSCs and are strong evidence of the existence of germline stem cells in mammalian ovaries after birth.

In addition, the study of FGSCs has been hampered by their low numbers and the lack of methods for isolation and identification, and little was known previously regarding the molecular mechanisms of FGSC self-renewal. Whereas many signalling pathways possibly maintain the biology of the stem cells, the PI3K-AKT pathway has been directly implicated in spermatogonial stem cell self-renewal, which is another stem cell that has germline potential. GDNF was the first molecule that was found to be involved in the selfrenewal of spermatogonial stem cell, and studies have shown that GDNF can activate the PI3K-AKT pathway to promote proliferation [23]. Some researchers recently found that retinoic acid stimulation of the PI3K/AKT/mTOR was required for the differentiation of spermatogonial stem cell [45]. Furthermore, previous studies have suggested that human SSCs can be cultivated for a long period and expanded whilst retaining an undifferentiated 


\section{Cellular Physiology Cell Physiol Biochem 2018;46:2114-2126 \begin{tabular}{l|l} 
DOI: 10.1159/000489452 & Ond Biochemistry 2018 The Author(s). Published by S. Karger AG, Basel \\
mww.karger.com/cpb
\end{tabular} \\ Wu et al.: Enrichment of Female Germline Stem Cells}

status via the activation of SMAD3 and AKT pathways [24]. It is well-known that the AKT pathway is involved in self-renewal of SSCs, but little is known regarding the effects of the AKT pathway on self-renewal of FGSCs. FGSCs and spermatogonial stem cells are similar in their proliferation and differentiation patterns $[28,46]$, which raises the possibility that the self-renewal mechanism may be similar between these two systems. It has been reported that the PI3K-AKT pathway is highly expressed in FGSCs, demonstrating that the PI3K-AKT pathway may participate in the self-renewal of FGSCs [46]. Furthermore, previous studies have shown that PI3K signalling plays a key role in controlling the growth of oocytes during the activation of primordial follicles in mice, and the activation of PI3K pathway accelerated the transformation of primordial follicles to primary follicles; these results have also been confirmed in POI patients $[47,48]$. The possible reason is that AKT activation may lead to proliferation of FGSCs and differentiation into primordial follicles. In this study, our results demonstrated that inhibition of the AKT pathway prevented FGSC proliferation. Treatment with an inhibitor (LY294002) of the PI3K pathway resulted in downregulation of the expression of proliferation markers, including c-Myc and PCNA. Moreover, several cell cycle regulators, known to regulate cell cycle progression during G1/S transition, were also detected. Cyclin A, cyclin B1 and cyclin D1 were downregulated in the LY294002 group. c-Myc plays a part in many biological functions, including cell proliferation, apoptosis, and differentiation [49]. Proliferating cell nuclear antigen (PCNA) is a distinct marker for cell proliferation [50], which was downregulated in the LY294002 group. In addition, our results revealed that AKT overexpression increased the proliferation of FGSCs as well as the mRNA expression of MVH, Oct-4, and the proportion of Gdf- $9^{+}$(specific marker of oocyte) cells also increased. These results demonstrate that the AKT pathway may regulate FGSC self-renewal. In future study, we will further explore the mechanism of FGSCs differentiation and try to induce FGSCs to differentiate into germ cells in vitro. It has already reported that many other stem cells could be successfully induced as germ cells. In 2003, a group headed by Hans R. Schöler reported that oocyte-like cells could be produced from mouse embryonic stem cells (ESCs) in vitro [51]. Ten years later, Katsuhiko et al. reported that they could get offspring from oocytes derived from in vitro primordial germ cells like cells (PGCLCs) in Mice [52]. In 2014, Kimura et al. demonstrated that PGCLCs were efficiently induced from mouse ESCs by ERK signaling inhibition [53]. And Wei Ge et al. first demonstrated that human skin-derived stem cells possessed the differentiation potential into germ lines without reprogramming into the iPS cell stage [54]. Other researchers have got germ cells from spermatogonial stem cells, mesenchyma stem cells and pancreatic stem cells [55-57]. These research results will promote the study of the differentiation of FGSCs.

In conclusion, through isolation, identification and functional verification of DA-FGSCs, we confirmed that differential adhesion was a feasible and simple method to establish an FGSC line. In addition, to the best of our knowledge, this report is the first to demonstrate that the AKT pathway regulates the FGSC self-renewal. Although there are some restrictions in our study, the outcome of this study may facilitate FGSC research, encourage more researchers to participate in the field of FGSCs and may resolve such problems as infertility and ovarian ageing.

\section{Acknowledgements}

This work was supported by grants from the National Natural Science Foundation of China (no. 81370469, 81671394 and 81701438) and Integrated Innovative Team for Major Human Diseases Program of Tongji Medical College, HUST (5001540038).

\section{Disclosure Statement}

The authors declare that they have no conflicts of interest regarding the contents of this article. 


\section{Cellular Physiology Cell Physiol Biochem 2018;46:2114-2126

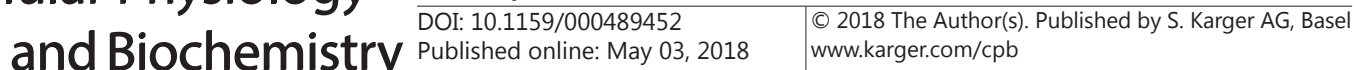

Wu et al.: Enrichment of Female Germline Stem Cells

\section{References}

1 Nakamura S, Kobayashi K, Nishimura T, Higashijima S, Tanaka M: Identification of Germline Stem Cells in the Ovary of the Teleost Medaka. Science 2010;328:1561-1563.

2 Kirilly D, Xie T: The Drosophila ovary: an active stem cell community. Cell Res 2007;17:15-25.

-3 Anderson LD, Hirshfield AN: An overview of follicular development in the ovary: from embryo to the fertilized ovum in vitro. Md Med J 1992;41:614-620.

$>4$ Borum K: Oogenesis in the mouse. A study of the meiotic prophase. Exp Cell Res 1961;24:495-507. Telfer EE, Gosden RG, Byskov AG, Spears N, Albertini D, Andersen CY, Anderson R, Braw-Tal R, Clarke H, Gougeon A, McLaughlin E, McLaren A, McNatty K, Schatten G, Silber S, Tsafriri A: On regenerating the ovary and generating controversy. Cell 2005;122:821-822.

6 Kerr JB, Duckett R, Myers M, Britt KL, Mladenovska T, Findlay JK: Quantification of healthy follicles in the neonatal and adult mouse ovary: evidence for maintenance of primordial follicle supply. Reproduction 2006;132:95-109.

7 Johnson J, Canning J, Kaneko T, Pru JK, Tilly JL: Germline stem cells and follicular renewal in the postnatal mammalian ovary. Nature 2004;428:145-150.

8 Zou K, Yuan Z, Yang Z, Luo H, Sun K, Zhou L, Xiang J, Shi L, Yu Q, Zhang Y, Hou R, Wu J: Production of offspring from a germline stem cell line derived from neonatal ovaries. Nat Cell Biol 2009;11:631-636.

9 Zhang C, Wu J: Production of offspring from a germline stem cell line derived from prepubertal ovaries of germline reporter mice. Mol Hum Reprod 2016;22:457-464.

10 Zhang H, Panula S, Petropoulos S, Edsgard D, Busayavalasa K, Liu L, Li X, Risal S, Shen Y, Shao J, Liu M, Li S, Zhang D, Zhang X, Gerner RR, Sheikhi M, Damdimopoulou P, Sandberg R, Douagi I, Gustafsson JA, Lanner F, Hovatta O, Liu K: Adult human and mouse ovaries lack DDX4-expressing functional oogonial stem cells. Nat Med 2015;21:1116-1118.

11 White YA, Woods DC, Takai Y, Ishihara O, Seki H, Tilly JL: Oocyte formation by mitotically active germ cells purified from ovaries of reproductive-age women. Nat Med 2012;18:413-421.

12 Xiong J, Lu Z, Wu M, Zhang J, Cheng J, Luo A, Shen W, Fang L, Zhou S, Wang S: Intraovarian Transplantation of Female Germline Stem Cells Rescue Ovarian Function in Chemotherapy-Injured Ovaries. Plos One 2015;10:e0139824.

13 Lu Z, Wu M, Zhang J, Xiong J, Cheng J, Shen W, Luo A, Fang L, Wang S: Improvement in Isolation and Identification of Mouse Oogonial Stem Cells. Stem Cells Int 2016;2016:2749461.

-14 Ding X, Liu G, Xu B, Wu C, Hui N, Ni X, Wang J, Du M, Teng X, Wu J: Human GV oocytes generated by mitotically active germ cells obtained from follicular aspirates. Sci Rep 2016;6:28218.

15 Telfer EE, Albertini DF: The quest for human ovarian stem cells. Nat Med 2012;18:353-354.

16 Oatley J, Hunt PA: Of mice and (wo)men: purified oogonial stem cells from mouse and human ovaries. Biol Reprod 2012;86:196.

-17 Zhang H, Zheng WJ, Shen Y, Adhikari D, Ueno H, Liu K: Experimental evidence showing that no mitotically active female germline progenitors exist in postnatal mouse ovaries. Proc Natl Acad Sci U S A 2012;109:12580-12585.

18 Hernandez SF, Vahidi NA, Park S, Weitzel RP, Tisdale J, Rueda BR, Wolff EF: Characterization of extracellular DDX4- or Ddx4-positive ovarian cells. Nat Med 2015;21:1114-1116.

19 Zarate-Garcia L, Lane SI, Merriman JA, Jones KT. FACS-sorted putative oogonial stem cells from the ovary are neither DDX4-positive nor germ cells. Sci Rep 2016;6:27991.

20 Lei L, Spradling AC: Female mice lack adult germ-line stem cells but sustain oogenesis using stable primordial follicles. Proc Natl Acad Sci U S A 2013;110:8585-8590.

21 Zhang H, Liu L, Li X, Busayavalasa K, Shen Y, Hovatta O, Gustafsson JA, Liu K: Life-long in vivo cell-lineage tracing shows that no oogenesis originates from putative germline stem cells in adult mice. Proc Natl Acad Sci U S A 2014;111:17983-17988.

22 Guo K, Li CH, Wang XY, He DJ, Zheng P: Germ stem cells are active in postnatal mouse ovary under physiological conditions. Mol Hum Reprod 2016;22:316-328.

-23 Arnhold S, Wenisch S: Adipose tissue derived mesenchymal stem cells for musculoskeletal repair in veterinary medicine. Am J Stem Cells 2015;4:1-12.

-24 Kreider BQ Messing A, Doan H, Kim SU, Lisak RP, Pleasure DE: Enrichment of Schwann cell cultures from neonatal rat sciatic nerve by differential adhesion. Brain Res 1981;207:433-444. 


\section{Cellular Physiology Cell Physiol Biochem 2018;46:2114-2126

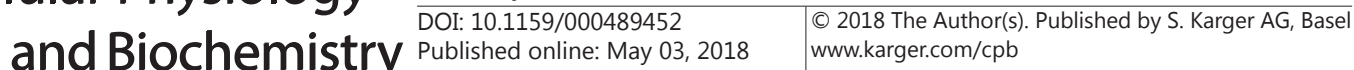 \\ Wu et al.: Enrichment of Female Germline Stem Cells}

25 Sato T, Katagiri K, Kubota Y, Ogawa T: In vitro sperm production from mouse spermatogonial stem cell lines using an organ culture method. Nat Protoc 2013;8:2098-2104.

26 Wang H, Shi L, Xiang J, Ding X, Luo H, Wang S, Wu J: Isolation, culture and transplantation of female germline stem cells from neonatal and prepubertal mice. Protocol Exchange DOI:10.1038/protex.2013.004.

-27 Liu J, Shang D, Xiao Y, Zhong P, Cheng H, Zhou R: Isolation and characterization of string-forming female germline stem cells from ovaries of neonatal mice. J Biol Chem 2017;292:16003-16013.

28 Li X, Ao J, Wu J: Systematic identification and comparison of expressed profiles of lncRNAs and circRNAs with associated co-expression and ceRNA networks in mouse germline stem cells. Oncotarget 2017;8:26573-26590.

29 Pacchiarotti J, Maki C, Ramos T, Marh J, Howerton K, Wong J, Pham J, Anorve S, Chow YC, Izadyar F: Differentiation potential of germ line stem cells derived from the postnatal mouse ovary. Differentiation 2010;79:159-170.

-30 Clermont Y: Kinetics of spermatogenesis in mammals: seminiferous epithelium cycle and spermatogonial renewal. Physiol Rev 1972;52:198-236.

31 Sato T, Katagiri K, Yokonishi T, Kubota Y, Inoue K, Ogonuki N, Matoba S, Ogura A, Ogawa T: In vitro production of fertile sperm from murine spermatogonial stem cell lines. Nat Commun 2011;2:472.

-32 Woods DC, Tilly JL: Adult human and mouse ovaries lack DDX4-expressing functional oogonial stem cells Reply. Nat Med 2015;21:1118-1121.

33 Yuan J, Zhang D, Wang L, Liu M, Mao J, Yin Y, Ye X, Liu N, Han J, Gao Y, Cheng T, Keefe DL, Liu L: No evidence for neo-oogenesis may link to ovarian senescence in adult monkey. Stem Cells 2013;31:2538-2550.

34 Hernandez SF, Vahidi NA, Park S, Weitzel RP, Tisdale J, Rueda BR, Wolff EF: Characterization of extracellular DDX4-or Ddx4-positive ovarian cells. Nat Med 2015;21:1114-1116.

-35 Parte S, Bhartiya D, Telang J, Daithankar V, Salvi V, Zaveri K, Hinduja I: Detection, Characterization, and Spontaneous Differentiation In vitro of Very Small Embryonic-Like Putative Stem Cells in Adult Mammalian Ovary. Stem Cells Dev 2011;20:1451-1464.

-36 Dunlop CE, Bayne RA, McLaughlin M, Telfer EE, Anderson RA: Isolation, purification, and culture of oogonial stem cells from adult human and bovine ovarian cortex. Lancet 2014;383:45-45.

37 Park ES, Woods DC, Tilly JL: Bone morphogenetic protein 4 promotes mammalian oogonial stem cell differentiation via Smad1/5/8 signaling. Fertil Steril 2013;100:1468-1475.

38 Zhou L, Wang L, Kang JX, Xie W, Li X, Wu C, Xu B, Wu J: Production of fat-1 transgenic rats using a post-natal female germline stem cell line. Mol Hum Reprod 2014;20:271-281.

39 Ye H, Li X, Zheng T, Hu C, Pan Z, Huang J, Li J, Li W, Zheng Y: The Hippo Signaling Pathway Regulates Ovarian Function via the Proliferation of Ovarian Germline Stem Cells. Cell Physiol Biochem 2017;41:1051-1062.

40 Li J, Zhou F, Zheng T, Pan Z, Liang X, Huang J, Zheng L, Zheng Y: Ovarian Germline Stem Cells (OGSCs) and the Hippo Signaling Pathway Association with Physiological and Pathological Ovarian Aging in Mice. Cell Physiol Biochem 2015;36:1712-1724.

-41 Terraciano P, Garcez T, Ayres L, Durli I, Baggio M, Kuhl CP, Laurino C, Passos E, Paz AH, Cirne-Lima E: Cell therapy for chemically induced ovarian failure in mice. Stem Cells Int 2014;2014:720753.

$\$ 42$ Woods DC, Tilly JL: Autologous Germline Mitochondrial Energy Transfer (AUGMENT) in Human Assisted Reproduction. Semin Reprod Med 2015;33:410-421.

43 Albertini DF, Gleicher N: A detour in the quest for oogonial stem cells: methods matter. Nat Med 2015;21:1126-1127.

44 Zou K, Hou L, Sun K, Xie W, Wu J: Improved efficiency of female germline stem cell purification using fragilis-based magnetic bead sorting. Stem Cells Dev 2011;20:2197-2204.

$\$ 45$ Busada JT, Chappell VA, Niedenberger BA, Kaye EP, Keiper BD, Hogarth CA, Geyer CB: Retinoic acid regulates Kit translation during spermatogonial differentiation in the mouse. Dev Biol 2015;397:140-149.

46 Zhang XL, Wu J, Wang J, Shen T, Li H, Lu J, Gu Y, Kang Y, Wong CH, Ngan CY, Shao Z, Zhao X: Integrative epigenomic analysis reveals unique epigenetic signatures involved in unipotency of mouse female germline stem cells. Genome Biol 2016;17:162.

47 Kawamura K, Cheng Y, Suzuki N, Deguchi M, Sato Y, Takae S, Ho CH, Kawamura N, Tamura M, Hashimoto S, Sugishita Y, Morimoto Y, Hosoi Y, Yoshioka N, Ishizuka B, Hsueh AJ: Hippo signaling disruption and Akt stimulation of ovarian follicles for infertility treatment. Proc Natl Acad Sci U S A 2013;110:17474-17479. 


\section{Cellular Physiology Cell Physiol Biochem 2018;46:2114-2126

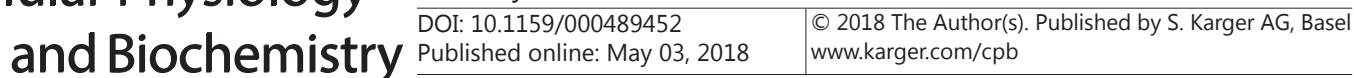

Wu et al.: Enrichment of Female Germline Stem Cells

48 Reddy P, Liu L, Adhikari D, Jagarlamudi K, Rajareddy S, Shen Y, Du C, Tang W, Hamalainen T, Peng SL, Lan ZJ, Cooney AJ, Huhtaniemi I, Liu K: Oocyte-specific deletion of Pten causes premature activation of the primordial follicle pool. Science 2008;319:611-613.

49 Luscher B: Function and regulation of the transcription factors of the Myc/Max/Mad network. Gene 2001;277:1-14.

50 Steger K, Aleithe I, Behre H, Bergmann M: The proliferation of spermatogonia in normal and pathological human seminiferous epithelium: an immunohistochemical study using monoclonal antibodies against Ki67 protein and proliferating cell nuclear antigen. Mol Hum Reprod 1998;4:227-233.

51 Hubner K, Fuhrmann G, Christenson LK, Kehler J, Reinbold R, De La Fuente R, Wood J, Strauss JF, 3rd, Boiani M, Scholer HR: Derivation of oocytes from mouse embryonic stem cells. Science 2003;300:1251-1256.

52 Hayashi K, Ogushi S, Kurimoto K, Shimamoto S, Ohta H, Saitou M: Offspring from oocytes derived from in vitro primordial germ cell-like cells in mice. Science 2012;338:971-975.

53 Kimura T, Kaga Y, Ohta H, Odamoto M, Sekita Y, Li K, Yamano N, Fujikawa K, Isotani A, Sasaki N, Toyoda M, Hayashi K, Okabe M, Shinohara T, Saitou M, Nakano T: Induction of primordial germ cell-like cells from mouse embryonic stem cells by ERK signal inhibition. Stem Cells 2014;32:2668-2678.

54 Ge W, Ma HG, Cheng SF, Sun YC, Sun LL, Sun XF, Li L, Dyce P, Li J, Shi QH, Shen W: Differentiation of early germ cells from human skin-derived stem cells without exogenous gene integration. Sci Rep 2015;5:13822.

55 Wang L, Cao J, Ji P, Zhang D, Ma L, Dym M, Yu Z, Feng L: Oocyte-like cells induced from mouse spermatogonial stem cells. Cell Biosci 2012;2:27.

-56 Li PZ, Yan GY, Han L, Pang J, Zhong BS, Zhang GM, Wang F, Zhang YL: Overexpression of STRA8, BOULE, and DAZL Genes Promotes Goat Bone Marrow-Derived Mesenchymal Stem Cells In vitro Transdifferentiation Toward Putative Male Germ Cells. Reprod Sci 2017;24:300-312.

57 Danner S, Kajahn J, Geismann C, Klink E, Kruse C: Derivation of oocyte-like cells from a clonal pancreatic stem cell line. Mol Hum Reprod 2007;13:11-20. 